\title{
De la tolerancia al reconocimiento mutuo: Programa de formación para una ciudadanía activa ${ }^{1}$
}

\author{
Pilar Folgueiras Bertomeu \\ Universidad de Barcelona
}

\begin{abstract}
This article describes the theoretical and methodological basis that is fundamental to the elaboration, application and evaluation of a training program for active citizenship specifically designed for women. The program, which is being developed under the auspices of the U niversity of Barcelona, is based in the methodology of participatory action research, which allows for the research subjects' participation in the entire process, including the evaluation. The research confirms the necessity of creating spaces for citizenship participation that foster the rights and responsibilities of participation, allow people to acquire the necessary citizenship competencies in order to have an active presence in the public sphere, and promote a type of civic identity based in dial ogue and in the value of solidarity.
\end{abstract}

Key words: Tolerance, recognition, training program, active citizenship, participatory evaluation

\section{RESUMEN}

Este artículo describe las bases teóricas y metodológicas que fundamentan la elaboración, aplicación y evaluación de un programa de formación para una ciudadanía activa específicamente diseñado para mujeres. El programa, que se desarrolla con el auspicio de la U niversidad de Barcelona, esta basado en la metodología de la evaluación participativa, la cual apuesta por la participación de las personas en todo el proceso. Los primeros datos de la investigación confirman la necesidad de crear y promover espacios de participación ciudadana donde se fomente el derecho y la responsabilidad de participar, se adquieran las competencias ciudadanas necesarias para que las personas puedan tener presencia activa en el espacio público, y se promueva un tipo de identidad cívica basada en el diálogo y en el valor de la solidaridad.

Descriptores: Tolerancia, reconocimiento, programa de formación, ciudadanía activa, evaluación participativa

\section{RÉSUMÉ}

Cet article décrit la base théorique et méthodologique qui est fondamentale à l'élaboration, l'application et l'évaluation d'un programme de formation à la citoyenn eté active conçu spécifiquement pour les femmes. Le programme qui est en voie de développement sous les auspices de l'université de Barcelone, est basé sur la méthodologie de la recherche-action participative, qui permet la participation du sujet de la recherche dans tout le processus, y compris l'éval uation. La recherche confirme la nécessité de créer des espaces pour la participation citoyenne qui favorisent les droits et responsabilités de la participation, qui permettent aux personnes d'acquérir les compétences de citoyenneté nécessaires pour exercer une présence active dans la sphère publique et qui promeuvent un type d'identité civique enraciné dans le dialogue et la valeur de solidarité.

Mots-cléfs: Tolérance, reconnaissance, programme de formation, citoyenneté active, évaluation participative 


\section{Introducción}

E l trabajo que se presenta en este artículo se inscribe dentro de una línea de investigación centrada en el estudio sobre identidad y educación para la ciudadanía que el GREDI (Grupo de Investigación en Educación Intercultural, Universidad de Barcelona), viene desarrollando desde hace cuatro años. D entro de esta línea de investigación, el presente artículo describe las bases teóricas y metodológicas que fundamentan la elaboración, aplicación y evaluación de un programa de formación para una ciudadanía activa, dirigido a mujeres que viven en H ospitalet de Llobregat². En la primera parte del artículo se presentan las últimas cifras de las que disponemos sobre el total de la población inmigrante residente en España, en Cataluña y en H ospitalet de Llobregat. En la segunda parte se realiza una aproximación al marco teórico de la investigación. En tercer lugar se presenta la propuesta de innovación: un programa de formación para una ciudadanía activa específicamente diseñado para mujeres, seguidamente se expone el proceso metodológico seguido y, finalmente, se presentan las primeras conclusiones de la primera fase del estudio.

\section{Contexto empírico de la investigación ${ }^{3}$}

Lasúltimas cifras sobre inmigración revelan como el Estado español, fruto de una etapa de prosperidad y bonanza económica, se ha convertido en un país de destino para muchos ciudadanos y ciudadanas que buscan realizar sus proyectos en un país que durante mucho tiempo ha sido un país de emigrantes.

D urante la década de los 80, han sido muchas las personas que han llegado y se han establecido en el Estado español, la mayoría de ellas procedente del continente 4 . Posteriormente, en los años 90, ha sido cuando se ha producido un cambio en el perfil de las personas inmigrantes que pasan a provenir, mayoritariamente, de África y Sudamérica. Asimismo, ha sido a partir del año 1997 cuando la inmigración en el Estado español ha empezado a crecer de una forma más intensa y acelerada (M arín, 2002).

Según los datos aportados por el Anuario Estadístico de Extranjería del M inisterio del Interior ${ }^{5}$, en el año 1999 la inmigración extranjera representaba en el Estado Español un 2\% de la población (801.416 personas). Centrándonos en Cataluña en ese mismo año, representaba un 3\% de la población con un total de 183.743 personas inmigradas. Esta cifra ha ido creciendo en los tres últimosaños. Así, en el año 2000, el total de personas inmigrantes ascendía a 214.996 y en el año 2001 a 280.167, esta última cifra representaba el 4,4\% de la población catalana.

D el total dela población inmigrante en C ataluña, el grupo más representativo es el colectivo procedente de África del N orte con un total de 95.959 personas, de las cuales 88.643 proceden de M arruecos. El segundo mayor contingente de personas es el que procede de Latinoamérica con un total de 64.396 personas. El tercer lugar lo ocupa el colectivo procedente de los países de la U nión Europea (UE) donde el total de la población inmigrante asciende a 51.242 personas. Seguidamente, se sitúan las personas que proceden de Asia (36.224), del resto de África (18.337) y del este de Europa (14.550). 
Si atendemos a la distribución por municipios y según los datos facilitados por el Anuario de Estadística del Ayuntamiento de H ospitalet de Llobregat', en el año 2001 el número de personas empadronadas en la ciudad de H ospitalet de Llobregat es de 251.735, de las cuales el 130.587 del total han nacido en Cataluña, 103.484 son de otras comunidades autónomas y el 17.664 son de origen extranjero7. Centrándonos en la distribución por lugares de procedencia de la población extranjera (17.664 personas), el colectivo mayoritario es el americano con un total de población de 10.671, seguido del africano donde la cifra asciende a 4.631. En tercer lugar, se sitúa el colectivo procedente del continente asiático con un total de 1.083 personas y final mente, se encuentran las personas procedentes de países de la Comunidad Europea (648) y del resto de países de Europa (631 personas).

La distribución gráfica de la población por lugar de procedencia en H ospitalet de Llobregat es la siguiente:

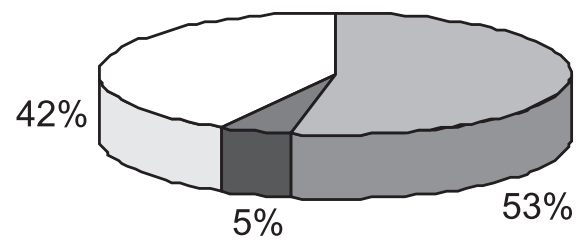

\author{
$\square$ Nacidos en Cataluña \\ $\square$ Nacidos fuera del Estado \\ Español \\ $\square$ Nacidos en otras \\ comunidades autónomas
}

Gráfico I: Distribución de la población por lugar de procedencia (año 2001).

Los datos proporcionados nos muestran como la inmigración está creciendo en C ataluña, convirtiendo a ciudades como H ospitalet de Llobregat en espacios cada vez más multiculturales y cosmopolitas. En estos nuevos espacios se están generando nuevas dinámicas ciudadanas que requieren, para su comprensión, ser pensadas desde esta diversidad de culturas así como desde el ejercicio de la ciudadanía y el espacio público (Borja, N el·lo y Vallès, 1998).

\title{
De la tolerancia al reconocimiento mutuo
}

Actualmente, el debate sobre quétipo de actitudes debieran fomentarse en la sociedad receptora de inmigrantes se ha centrado en una reconceptualización de la tolerancia (Bartolomé, 2002a), "virtud indiscutible de la democracia" (Camps, 1994: 96). Esta reconceptualización teórica pasa por la aceptación resignada de la diferencia con el fin de mantener la paz (C amps, 1994), actitud que es entendida como una forma de reprimir el rechazo y tolerar lo que nos incomoda, "es como si le dijéramos a alguien tolero tus creencias absurdas y tus actos sin sentido" (Berlin, 1988, citado en Camps, 1994: 93); hasta la admisión entusiasta de la diferencia, actitud que, coincidiendo con Walzer (1998), consideramos ya no sería tolerancią: "¿Cómo se puede decir que tolero lo que apruebo?" (Walzer, 1998: 26).

La aceptación resignada de la diferencia es causa de prácticas de tolerancia pasiva 9 , que se caracterizan por una fal ta de comunicación eintercambio entre las diferentes culturas que comparten un mismo espacio (Bauman, 2001). 
Un claro símbolo de este tipo de prácticas, en el Estado español, lo constituye el Ejido, uno de los municipios con mayor renta per cápita y con mayor índice de inmigración que, en los últimos años, ha protagonizado un fuerte crecimiento económico gracias a la explotación a la que se han visto sometidas un gran número de personas inmigrantes y a la indiferencia continuada de una parte de la ciudadanía y de algunas instituciones que, conocedoras de la violación de los $\mathrm{D}$ erechos $\mathrm{H}$ umanos que allí se estaba cometiendo, han permanecido en silencio durante más de diez años. Estas prácticas de tolerancia pasiva han sido el caldo de cultivo de las manifestaciones racistas y xenófobas que han tenido lugar durante los tres últimos años en este municipio, cuando los colectivos perjudicados han empezado a denunciar las situaciones de explotación que estaban sufriendo.

0 tro ejemplo de tolerancia pasiva nos lo proporciona el primer pacto de convivencia que en el Estado español se ha firmado entre un ayuntamiento y una comunidad musulmana de un pueblo de Barcelona. Léamos, hace unos meses, en una nota de prensa "el acuerdo recoge la creación de espacios de interrelación entre ciudadanos autóctonos einmigrantes, el papel mediador del ayuntamiento, la integración de los niños en la escuela, la igualdad de las mujeres, la inserción social de los nuevos ciudadanos, los modelos de convivencia y los principios democráticos que rigen la vida social del país deacogida" (La Vanguardia, domingo 9 de junio 2002). El acuerdo pone en evidencia como esta voluntad de convivencia, diálogo e intercambio se realiza al margen de las características de los nuevos llegados. Como muy acertadamente explica Amin M aalouf en su libro las "identidades asesinas", este tipo de prácticas son el resultado de entender que la sociedad de acogida es una página ya escrita, donde no cabe revisar, añadir o cambiar ninguno de los principios y val ores que la sustentan.

Por otro lado, también es importante señalar como este tipo de tolerancia pasiva puede ser fruto de la aceptación incondicional de cualquier tipo de diferencia, el tan famoso dicho "todo vale". Este tipo de actitud puede sumirnos en un fuerte relativismo donde se acaben aceptando y justificando actitudes y maneras de hacer en las que se están violando los $\mathrm{D}$ erechos $\mathrm{H}$ umanos de las personas.

Los ejemplos comentados nos avisan sobre la necesidad de avanzar hacia planteamientos recíprocos basados en el reconocimi ento mutuo (Taylor, 1994) entre las diferentes culturas. No pueden establecersejerarquías entre culturas ${ }^{10}$ (Panikkar, 2000), ni distinciones entre culturas de primera y de segunda categoría ${ }^{11}$ (Cortina, 1998) actitudes que única mente provocan situaciones de injusticia y desmotivación por la acción colectiva.

Llegar a ejercer prácticas de reconocimiento mutuo, supondría la consolidación de una ética ciudadana (Cortina, 1998), que entiende el hecho de que en una misma sociedad vivan diferentes personas con culturas diferentes como una oportunidad para crecer, tanto en el ámbito individual como social. Siguiendo a Cortina (1998) esta ética consistiría en:

- Posibilitar la adhesión a identidades culturales diversas ${ }^{12}$.

- No tener como meta el recrearse en la diferencia por la diferencia y distinguir entre posiciones respetables aunque uno no esté de acuerdo con ellas y posiciones no respetables. 
- El respeto activo hacia las diferentes culturas, actitud que está relacionada con el respeto a las identidades.

- La comprensión de otras culturas como elemento indispensable para comprender la propia.

D entro de esta ética ciudadana también cabria añadir como elemento imprescindible la igualdad de oportunidades de todas las personas al margen de sus culturas de origen. Llegar a esta ética ciudadana supondría el desarrollo de actitudes entusiastas hacia la diferencia, posicionamiento teórico que ya enunciábamos al inicio de este apartado.

\section{La participación ciudadana: una vía de respuesta en sociedades multiculturales}

Son muchos los autores y las autoras que ven en la participación ${ }^{13}$ una llave para educar en la diversidad (M ouffe citado en Pagé, 1997); promover la formación de vínculos entrelas personasasí como, entreellasy la comunidad en la que participan (H abermas, 1998; Pateman, 1970); mejorar las condiciones de vida democrática (Pateman, 1970) y desarrollar actitudes positivas hacia uno mismo (C ortina, 1998; Pateman, 1970). Pero, ¿cómo debería ser esta participación? ¿Q uéaspectos se deberían tener en cuenta en la formación para la participación? ¿D ónde se debería ejercer esta participación? Para contestar a estos interrogantes nos remitiremos a los que considero los principales elementos de la participación.

\section{La participación ciudadana: un derecho básico de ciudadanía}

El derecho de participación ${ }^{14}$, en el mundo actual, debeser un elemento básico del estatus del ciudadano (Cortina, 1998). Si esta dimensión no está resuelta, difícilmente las personas se sentirán miembros de esa comunidad. Por tanto, el primer paso para que la integración de las personas sea posible es que sean reconocidas legal mente como ciudadanas. El reconocimiento de su estatus legal, les permitirá participar legal mente en la sociedad.

\section{La participación ciudadana: una responsabilidad} social de la ciudadanía

Las sociedades actuales están experimentando numerosas transformacione ${ }^{15}$ que sin duda alguna pasan por la necesidad de ampliar el concepto de participación ciudadana entendiéndolo no únicamente como un derecho, sino también como una responsabilidad social: "en la acción que ayuda a los procesos de transformación democrática, es tan importante (einseparable) la formación para la participación como para la responsabilidad social" (Luque D ominguez, 1995: 51).

Las ciudadanas y los ciudadanos del siglo XXI deberemos comprender que la implicación activa es necesaria si queremos ser protagonistas en los procesos de cambio y si queremos que éstos se realicen desde su dimensión educativa y social. Además, participar activamente en la sociedad contribuirá a que todos los derechos, incluidos los de participación, lleguen a todas las personas (C amps, 1998). 
La participación ciudadana: un instrumento de "empowerment"

La participación ha sido tradicionalmente, y puede continuar siéndolo, un elemento de dualización ${ }^{16}$ de la sociedad (G aventa-Valderrama, 1999) que divide a la población entre los que participan y los que no lo hacen. D entro de esta segunda categoría, el género, la cultura y la clase social han sido y son elementos clásicos de exclusión ${ }^{17}$.

Para luchar contra esta exclusión, la participación debe constituirse como un instrumento de empowerment para que las personas, sobre todo aquellas que tradicionalmente han sido excluidas, adquieran las competencias ciudadanas necesarias para poder tener presencia activa en el espacio público (M archioni, 1999; U rgenson-Kember, 1997). Así, la participación entendida como empowerment crea oportunidades para que todos los miembros de la comunidad contribuyan activamente en el proceso de desarrollo y búsqueda de equidad.

La participación ciudadana: un elemento generador de identidad cívica

La participación promueve la formación de vínculos cívicos entre las personas así como entre ellas y la comunidad en la que participan (H abermas, 1998; Pateman, 1970). Por tanto, siguiendo a estos autores, se puede afirmar que las dinámicas participativas son generadoras de un tipo de identidad cívica (H érbert, 1997) que nada tiene que ver con la pertenencia a una comunidad prepolítica en la que la integración se alcanza por medio de la descendencia, la tradición y el lenguaje común, sino quees fruto del diálogo ${ }^{18}$, las opiniones, el intercambio, etc., que se genera entre personas que participan en un mismo espacio.

\section{La participación ciudadana de mujeres ${ }^{19}$ : del espacio privado al espacio público}

Véamos en el apartado anterior como el género ha sido uno de los elementos clásicos de exclusión de la participación (Lister, 1997; Pateman, 1970; Siim, 2000). Tradicionalmente, este colectivo ha sido privado de participar en el espacio público en favor de los hombres, quienes sí que han gozado de plena autonomía y libertad para autorrealizarse e influenciar en él. Esta discriminación se refleja ya en la separación entre la esfera privada y la esfera pública, separación que ha ido acompañada de una caracterización de cada una de ellas en función del género y a la que Pateman (1970) ha denominado separación patriarcal. Entre sus principales características Lister (1997) señala: 
Tabla I: La Participation Ciudadana

\begin{tabular}{ll}
\hline ESFERA PÚBLICA, HOMBRE, CIUDADANO20 & ESFERA PRIVADA, MUJER, NO CIUDADANA \\
\hline Abstracto y mental & Particular y sentimental \\
\hline Racional & Emocional \\
\hline Imparcial y preocupado por los & Parcial y preocupada por los temas de interés \\
temas de interés público & privado y doméstico \\
\hline Independiente, activo y fuerte & D ependiente, pasiva y débil
\end{tabular}

(Adaptado de Lister, 1997: 69)

En la misma línea Toren (1999) reafirmará como las características de los hombres son la racionalidad, la objetividad, el pensamiento abstracto y la competitividad. Asimismo, relacionándolo con el tema de la inmigración, la discriminación que sufren las mujeres resulta mucho más acusada cuando éstas proceden de otras culturas, de manera que la dimensión cultural otorga también, a la mujer, un factor añadido de marginación. "El género es solo un elemento en esta tensión (...) La posición de las mujeres como ciudadanas vendrá mediatizada por otrosfactores como la etnicidad" (U rgenson \& Kember, 1997:4).

Superar esta discriminación requiere del empowerment de las mujeres mediante su participación activa en el espacio público, retomando palabras de M archioni (1999) que la integración de la mujer en el espacio público debe ser un proceso intrínseco en cualquier intervención comunitaria.

A partir de la relación entre los diferentes elementos que han ido apareciendo y de la propia dinámica participativa se genera un modelo de formación para una ciudadanía activa; amplio, múltipley dinámico. Gráficamente, el modelo propuesto es el siguiente:

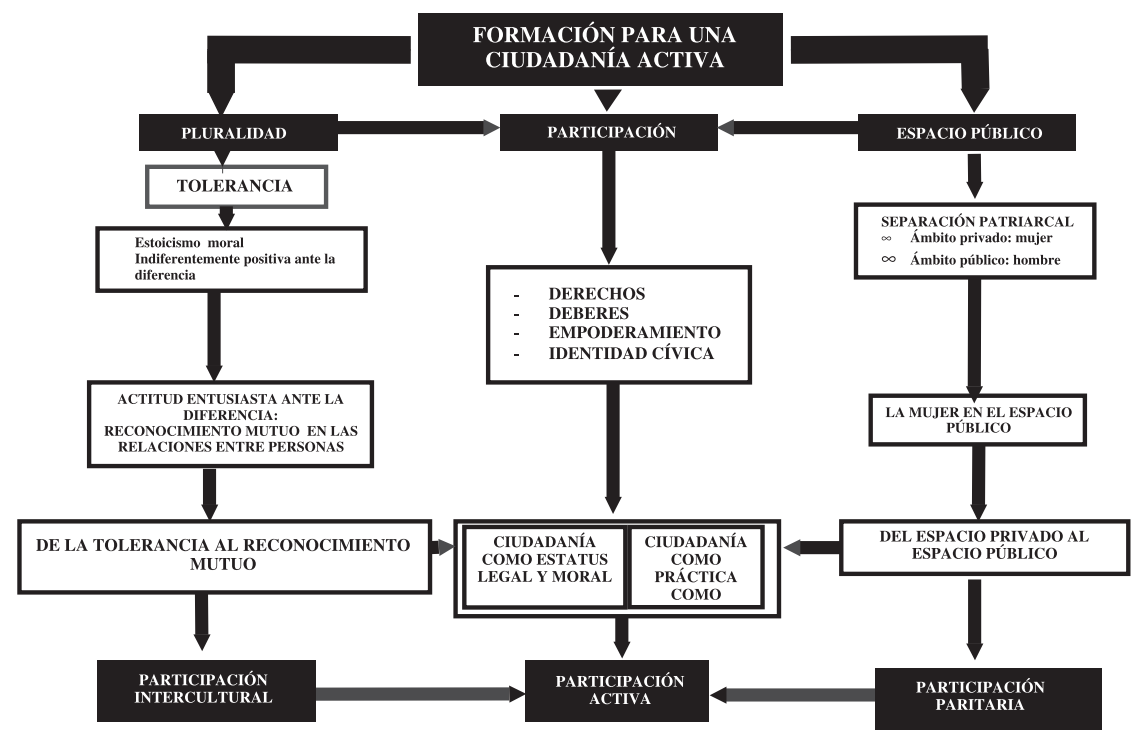

Gráfico II: Ampliación del modelo de formación para una ciudadania activ 
En el modelo de formación para una ciudadanía activa, se aprecian tres dimensiones claves de la participación:

- Participación activa, generada a partir de la interrelación entre los cuatro elementos claves de la participación; derecho (ciudadanía como estatus legal), responsabilidad social (ciudadanía como estatus moral), "empowerment" eidentidad cívica (ciu-dadanía como práctica).

- Participación intercultural, generada a partir de relaciones basadas en el reconocimiento mutuo entre personas que participan activamente en un mismo espacio.

- Participación paritaria, superación de la desigual dad de género a partir de la paticipación activa de las mujeres.

Todas estas dimensiones así como las relaciones que se establecen entre todos los elementos que han ido apareciendo hacen que el modelo propuesto sea:

- Amplio, dado que el ciudadano como sujeto de derechos únicamente es hoy insuficiente (C abrera, 2002).

- Múltiple, porque debe incluir los diferentes elementos que han ido apareciendo (Bartolomé, 2002b).

- Dinámico, porque todos los elementos están en interacción y son interdependientes (Bartolomé, 2002b).

En esta concepción amplia, múltiple y dinámica de ciudadanía, la educación pasa a ser una parte integral de todo el proceso (H eater, 1999).

\section{Propuesta de intervención: un programa de formación para una ciudadanía activa ${ }^{21}$}

La conceptualización y la articulación teórica elaborada sobre el constructo de la participación así como la aproximación diagnóstica realizad $a^{22}$, nos avisan sobre la necesidad de fomentar espacios centrados en la participación de las mujeres. Con tal fin, se desarrolla una propuesta de intervención consistente en un programa de actividades centradas en la formación para una ciudadanía activa. Este programa constituye la base del proceso de evaluación participativa que actual mente se ha iniciado.

\section{Objetivos generales del programa}

La propuesta persigue tres objetivos generales que coinciden con los elementos claves de la participación (ver gráfico II):

- Favorecer el derecho y la responsabilidad de participar en un grupo de mujeres que viven en una misma ciudad; el derecho dado que se promueve un espacio de participación donde todas las personas pueden participar al margen de su situación legal. La responsabilidad porque se intenta sensibilizar a las personas participantes sobre la necesidad de participar para transformar y mejorar tanto la 
vida personal como la social.

- Favorecer el empowerment por parte de un grupo de mujeres que viven en una misma ciudad; se incide, por un lado, en las habilidades organizativas, dialógicas, en los elementos de juicio crítico, en el sentimiento de autoestima y en la creatividad y, por el otro, en la búsqueda, la sel ección y la organización de la información mediante la utilización de herramientas informáticas.

- Promover la identidad cívica entre un grupo de mujeres que viven en una misma ciudad; se trabaja, fundamentalmente, el conocimiento de la comunidad y las relaciones de reconocimiento mutuo basadas en el diálogo y en el val or de la solidaridad.

\section{Organización del material}

El material se desarrolla a partir de cinco bloques temáticos conociéndonos, organizándonos; analizando el problema; buscando recursos y realizando alguna acción integrados por diferentes actividades.

\section{Tabla II: Cineo BloquesTemáticos}

\begin{tabular}{ll}
\hline $\begin{array}{l}\text { El primer bloque: } \\
\text { Conociéndonos }\end{array}$ & $\begin{array}{l}\text { Recoge actividades para trabajar las características, las habilidades } \\
\text { personales, etc., de las personas participantes. }\end{array}$ \\
\hline El segundo bloque: & Recoge actividades centradas en la organización ${ }^{23}$ del grupo y en \\
Organizándonos & $\begin{array}{l}\text { algunas experiencias participativas donde, gracias a la organi- } \\
\text { zación de las personas, especialmente de las mujeres, se han con- } \\
\text { seguido importantes transformaciones individuales y/o sociales. }\end{array}$ \\
\hline
\end{tabular}

\section{El tercer bloque: Analizando ed problema}

Recogen propuestas de actividades centradas en estrategias de análisis para profundizar e intentar dar respuesta a las necesidades delas personas participantes.

\section{El cuarto bloque:}

\section{Buscando recursos}

\section{El cuarto bloque:}

\section{Realizando alguna acción}

Cada uno de los bloques temáticos esta integrado por diferentes actividades que responden a los tres objetivos señalados anteriormente y constituyen en sí mismas una herramienta diagnóstica para conocer los valores, sentimientos, actitudes etc. de las participantes en relación con las dimensiones fundamentales vinculadas al ejercicio de la participación.

Si bien el programa puede responder a esta secuenciación por bloques temáticos, dependiendo de las características de las participantes (grado de cohesión grupal, disponibilidad horaria, etc.) se cambiará el orden de las actividades, se flexibilizará de acuerdo a sus propuestas e intereses y se realizaran unas u otras del total que se proponen para cadabloque. 


\section{La evaluación de programas desde una perspectiva participativa}

En la propuesta de intervención se sigue una metodología de trabajo caracterizada por los rasgos más relevantes que distinguen a la evaluación partici pativa de otros métodos evaluativos. Recientemente, Bartolomé y C abrera (2000) han realizado una interesante sistematización de las nuevas tendencias en la evaluación de programas de educación multicultural y en su aportación se pone de manifiesto la utilidad y adecuación de los enfoques interpretativos y críticos en este ámbito, fundamental mente, del modelo participativo y otros estrechamente asociados a él (Folgueiras \& Sandín, 2003).

La evaluación participativa va más allá de considerar una investigación sobre los efectos de la intervención y apuesta por profundizar en los procesos de cambio y en la participación de las personas en el desarrollo de la intervención. Como señalan Carr y Kemmis (1988), entre otros, no es posible contentarse con resolver problemas mediante la aplicación de soluciones elaboradas al margen de los que participan en la práctica educativa. Asimismo, este enfoque evaluativo plantea al gunos el ementos clave distintivos de la investigación-acción participativa como son:

- Las necesidades se negocian colectivamente entre todas las personas participantes.

- Todas las tareas reflexivas y de acción van acompañada siempre de procesos formativos.

- La investigación tiene una finalidad claramente transformadora de la realidad que viven quienes participan en ella.

- Se elaboran documentos y se organizan sesiones de devolución informativa.

Insistir en que la evaluación participativa no sólo pretende la aplicación, adaptación y evaluación de un programa o materiales determinados de forma consensuada y participativa, sino que incorpora igualmente los cambios y transformaciones que el equipo de trabajo experimenta con relación a sus pensamientos y actitudes, la reflexión sobre las prácticas educativas y la formación adquirida a través del proceso.

Partiendo de estas premisas el proceso metodológico que se está siguiendo consta de tres fases:

1. Evaluación participativa inicial24; se realiza un diagnóstico en la organización ${ }^{25}$ en la que se lleva a cabo la innovación con el fin de; conocer la viabilidad del programa en el contexto en el que se aplica; detectar las necesidades expresadas y sentidas en relación al ejercicio de la participación; formar el equipo de trabajo y el grupo de mujeres que participan directamente en la innovación y; crear la comisión de seguimiento que acompaña todo el proceso de investigación.

2. Evaluación participativa de la aplicación; hace referencia a la aplicación y a la progresiva evaluación del programa de formación para una ciudadanía activa que, siguiendo los fundamentos teóricos de la evaluación participativa, se realiza conjuntamente con todas las personas que participan y tiene como objetivo principal la validación interna y externa del programa. Para ello, de manera sistemática y tras la aplicación de 
cada una de las actividades y dinámicas, el equipo de trabajo real iza reuniones formativas y, puntualmente, se realizan encuentros con la comisión de seguimiento.

3. Evaluación participativa de los resultados; hace referencia al proceso seguido así como a los resultados obtenidos y tiene como objetivos principales; conocer la incidencia del programa en todas las personas participantes y en la organización en la que se aplica y; conocer las posibilidades que ofrece el programa para futuras aplicaciones del

Gráficamente, el proceso metodológico completo que se está siguiendo queda recogido en el siguiente cuadro:

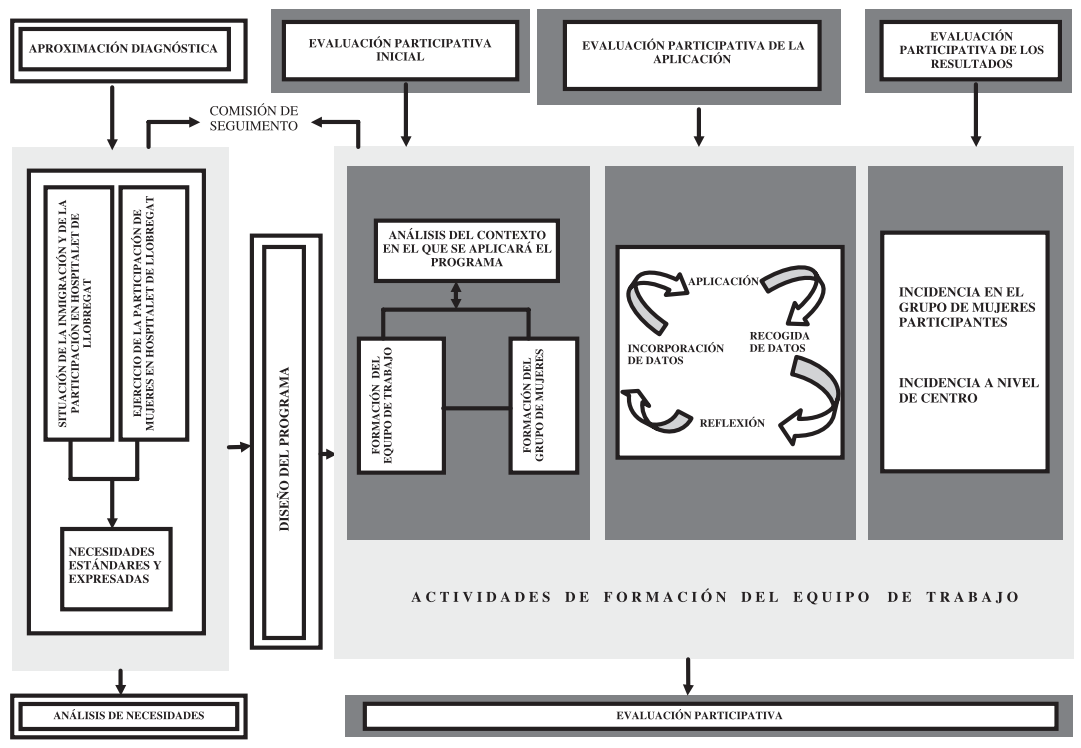

Gráfico III: M odelo metodológico

\section{Población/muestra de la evaluación participativa}

El programa de formación para una ciudadanía activa va dirigido a mujeres que viven en H ospitalet de Llobregat y está siendo aplicado en la Fundación A kwava. La muestra está formada por 33 mujeres que viven en esta ciudad y que proceden de diferentes lugares. Concretamente el $42 \%$ procede de M arruecos; el $15 \%$ de Ecuador; el $15 \%$ de República D ominicana; el 9\% de la República D emocrática del Congo; el 9\% de Estado Español; el 6\% deBolivia; el 3\% deCamerún y el 3\% restante de Líbano. 


\section{Primeras conclusiones de la primera fase de la evaluación participativa}

En apartados anteriores se han presentado brevemente algunos aspectos del marco teórico y metodológico que fundamentan el contenido y la puesta en práctica de la propuesta de intervención. A continuación me referiré, brevemente, a los primeros resultados que hemos obtenido en la primera fase de la evaluación participativa (ver gráfico III).

Durante la evaluación participativa inicial, se han realizado entrevistas semiestructuradas y dinámicas participativas a las 33 mujeres que integran la muestra. El análisis de contenido de las respuestas que dieron nos han ofrecido una visión de sus percepciones, creencias y actitudes respecto a los elementos de la participación.

Gráficamente, las principales categorías de análisis que se han utilizado para registrar, interpretar y analizar la información expresada por las mujeres son: ${ }^{26}$

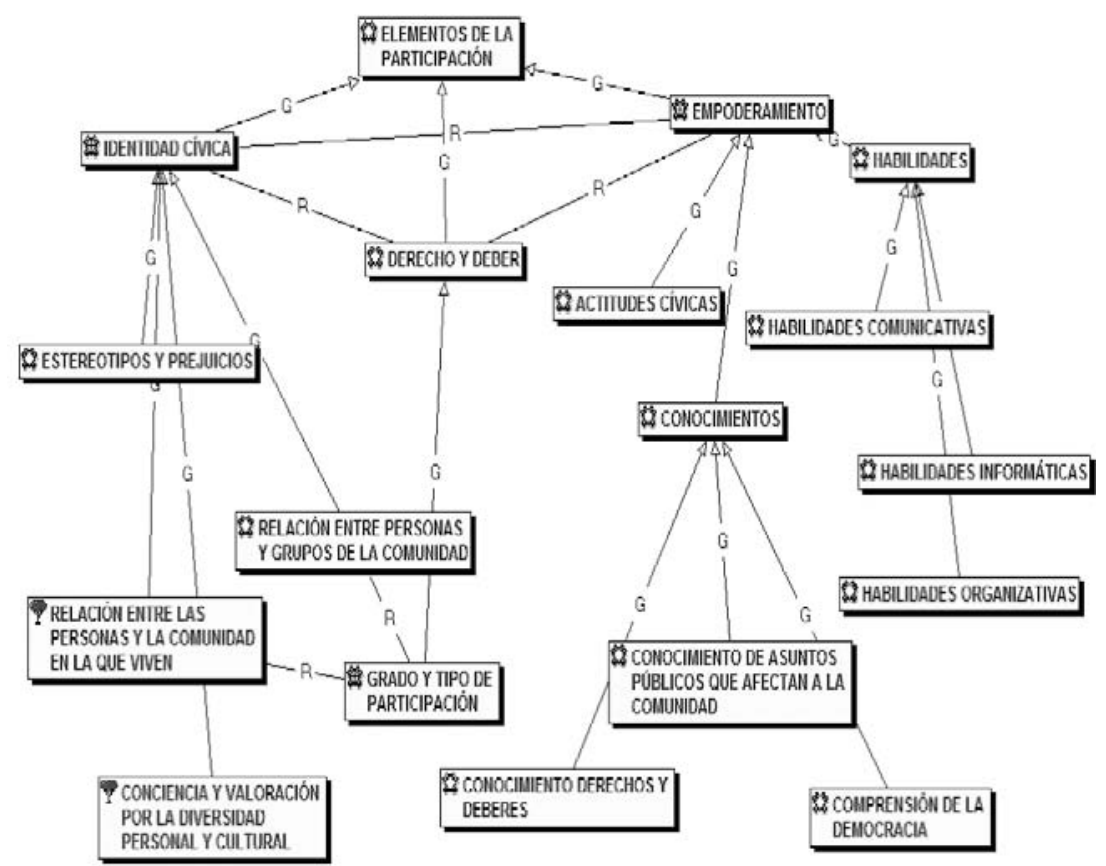

G ráfico IV: Relaciones entre las principales categorías de análisis ${ }^{27}$

Con relación al derecho y a la responsabilidad de participar, la información anal izada ha permitido detectar una clara y evidente diferenciación entre el tipo de participación en las elecciones que ejercían las mujeres en sus países de origen y que ejercen, ahora, en su nuevo país de acogida. Esta diferenciación es fruto de las barreras legales que existen y que impiden a las personas inmigrantes que no tienen la nacionalidad española disfrutar de este derecho de ciudadanía; por tanto vemos como las relaciones legales que se 
establecen entre trabajo formal, nación y ciudadanía están forjando un modelo de exclusión donde las personas no tienen derecho a participar en cuestiones que hacen referencia a los asuntos públicos. Así, del total de las mujeres entrevistadas ${ }^{28}$, todas, excepto ocho, manifestaron haber ejercido su derecho al voto cuando vivían en sus países de origen. Con relación a las ocho personas que no lo ejercían los motivos son, en dos de los casos, porque no tenían la mayoría de edad necesaria para poder ejercer este derecho, en otros tres por problemas políticos en sus países de origen y, en los tres restantes por desmotivación y desconfianza hacia sus representantes políticos

Contrariamente, esta falta de confianza que manifiestan estas tres mujeres no se da en otras maneras de participar. Así, cuando se les pregunta sobre su participación en las organizaciones e instituciones, ninguna de las entrevistadas manifiesta desconfianza y todas señalan como elementos principales que impiden su participación la falta de tiempo e información. Se pone en evidencia como una de las barreras que tradicionalmente han imposibilitado la participación de algunas personas sigue siendo las barreras organizativas. Especialmente, cuando se trata de mujeres, quienes continúan teniendo dificultades para participar a causa, principal mente, de los horarios que siguen estableciéndose, en muchas ocasiones, sobre los horarios masculinos (M archioni, 1999).

Respecto al segundo elemento generador de dinámicas participativas que anteriormente enunciábamos: Ia identidad cívica. En las respuestas que dan las mujeres, podemos advertir que no existe una relación estrecha entre ellas y la comunidad en la que viven, entendiendo por comunidad el barrio y la ciudad de H ospitalet de Llobregat. Prueba de ello es que la mayoría de mujeres, a pesar de mostrarse interesadas, no participan en las organizaciones, en las asociaciones, en las instituciones etc. ni de su barrio ni de su ciudad. Asimismo, no se relacionan ni con la población autóctona ${ }^{29}$, ni con personas pertenecientes a otros colectivos. A pesar de ello, en general manifiestan una actitud positiva y abierta hacia la mayoría de colectivos y personas. No en vano, todas las mujeres expresan sentirse satisfechas de que en su barrio vivan personas de diferentes culturas.

Por otro lado, las mujeres que llevan más tiempo viviendo en esta ciudad, a pesar de manifestar tener una actitud positiva hacia otros colectivos, creen que la inmigración se debería frenar ya que sino, en un futuro, no habrá trabajo para todas las personas. Esta reflexión pone en evidencia como, en muchas ocasiones, el rechazo hacia la personas migradas responde a un problema fundamentalmente económico así como, a la preocupación o miedo por perder lo que se ha conseguido. En palabras de Bilbeny (1995) se trataría de una actitud de xenomiedo.

Con relación al tercer elemento: el empowerment ciudadano. Las mujeres acusan una falta de conocimiento de sus derechos y responsabilidades e incluso algunas de las entrevistadas que se encuentran en situación irregular, manifiestan no saber cuál es el proceso legal que deberían seguir para regularizar su situación. También, a través de las entrevistas y las dinámicas participativas se puede ver que las mujeres conocen y expresan con claridad cuales son sus principales problemas; sin embargo, desconocen las vías y los canales de participación que podrían ayudarlas a mejorar su situación. De ahí que propongan organizar acciones centradas en sus problemas y necesidades que en muchos casos son las mismas y que les permitiría, por tanto, establecer objetivos comunes entre todas ellas. 
También, a partir de sus respuestas se pueden ver el gran número de barreras organizativas, legales y sociales que las mujeres se encuentran a la hora de participar, aspecto que, evidentemente, les desmotiva y les impide implicarse activamente en el barrio y en la ciudad en la que viven.

A partir de los datos expuestos y a modo de conclusión, se puede mencionar que la mayoría de personas entrevistadas (85\%) están interesadas en conocer los mecanismos de participación que existen en la ciudad de H ospitalet de Llobregat. M ás de la mitad de las entrevistadas (73\%) manifiestan estar interesadas en conocer a personas que viven en su misma ciudad y, un 59\% expresan querer conocer más aspectos sobre sus derechos y responsabilidades como ciudadanas de H ospitalet de Llobregat.

Por otro lado, durante esta primera fase de la eval uación participativa, también se ha constituido el equipo de trabajo integrado por una trabajadora social, una profesora, una alumna de educación social de la fundación y por mí misma. Semanalmente, el equipo se reúne para planificar cada una de las sesiones y para abordar conjuntamente todos los problemas que se derivan de la puesta en práctica de la innovación. Cabe señalar el alto grado de motivación del equipo de trabajo así como la importancia del proceso formativo que se sigue en cada reunión.

También se ha creado una comisión de seguimiento formada por el equipo de trabajo, por algunas de las mujeres participantes y por una persona vinculada al campo de la política y con representatividad en el ayuntamiento del municipio.

Todos los datos expuestos en este apartado así como el contenido de las reuniones con el equipo de trabajo nos avisan sobre la importancia y la necesidad de crear espacios centrados en la participación donde; se potencien el conocimiento de derechos y responsabilidades, el conocimiento de mecanismos de participación, etc. y, sefomenten espacios de diálogo en los que las personas se puedan conocer entre ellas y puedan hacer oír sus voces como ciudadanas de la ciudad en la que ahora viven.

\section{Notas}

1. El trabajo que se presenta forma parte de la tesis doctoral inscrita en el D epartamento de M étodos de Investigación y D iagnóstico en Educación, U niversidad de Barcelona.

2. H ospitalet de Llobregat es una ciudad contigua a Barcel ona y perteneciente a su área metropolitana.

3. La totalidad del trabajo que se está realizando incluye dos partes: análisis de necesidades y evaluación participativa, no obstante, en este artículo únicamente se hace referencia a la segunda parte: evaluación participativa.

4. La entrada del Estado español en la U nión Europea y la posterior adhesión en 1994 al acuerdo de Schengen, regulador de la libre circulación de personas en la U nión Europea, propició la entrada de un gran contingente de personas procedentes del continente europeo.

5. La consulta de los datos se ha realizado en la página W EB: http://www.ine.es/

6. Los datos han sido extraídos de la página W EB del Ayuntamiento de H ospitalet de Llobregat: http://www.l-h.es/anuari2001

7. Se constata progresivamente un aumento de residentes de natural eza extranjera, que en los datos del padrón municipal no quedan reflejados a causa de la situación de irregularidad que padece una gran parte de la población (Ayuntamiento de H ospitalet, 2000). 
8. La tolerancia acostumbra a implicar jerarquía, hay un al guien que tolera y un alguien tolerado. Las relaciones que se establecen no son, pues, horizontales (C ruz, 1998).

9. D iferentes estudios coinciden en distinguir dos tipos de tolerancia: la activa y la pasiva (Cruz, 1998).

10. Panikkar (2000) utiliza el concepto de interfecundación cultural para referirse a las relaciones simétricas entre culturas.

11. Gadamer, utiliza el concepto de fusión de horizontes para explicar como una cultura no es mejor que otra a priori sino cuando las restantes culturas se sienten obligadas a coger elementos de ella para responder a los retos sociales (citado en Cortina 1998).

12. M assot (2003) fundamenta el modelo de múltiple pertenencia, en el cual las personas construyen su identidad a partir del reconocimiento de sus múltiples pertenencias. Esto les permite funcionar adecuadamente en distintos ambientes socioculturales, siendo capaces de comprender, apreciar y compartir los valores, símbolos e instituciones de varias culturas.

13. La participación es un elemento básico en la actual conceptualización de ciudadanía. No en vano, se insiste en que la ciudadanía no es únicamente un estatus jurídico sino que es sobre todo una práctica ciudadana, un ejercicio activo que vincula al ciudadano con la comunidad. En palabras de H enderson-Salmon (1998)."A hora nosotros vamos hacia una versión más fuerte de la ciudadanía que está centrada en la participación" (Pág. 13).

14. La participación como derecho político se al canza en el siglo XIX y pasa a convertirse junto con los derechos civiles (siglo XVIII) y los derechos sociales (siglo XX) en el modelo de ciudadanía social conceptualizado por M arshall (1950), quien concibe al ciudadano como aquel que goza de unos derechos civiles (libertades individuales), derechos políticos (participación política) y derechos sociales (trabajo, educación, vivienda, salud etc.).

15. Entre los cambios y las transformaciones social es que están aconteciendo en las sociedades actuales, Bartolomé (coord.) (1999) señala: el proceso de globalización; la crisis de los Estados de Bienestar y el aumento persistente del desempleo; la multiculturalidad o la pluralidad de la sociedad actual; el incremento de los procesos migratorios; el cuestionamiento del Estado-nación; la superación del concepto de igualdad de oportunidades por el de equidad, las dificultades experimentadas en Europa para el desarrollo creciente de una identidad europea; la redefinición "femenina" del concepto de ciudadanía y la necesidad de un desarrollo sostenible.

16. En una investigación realizada por el CREA (1995), se evidencia como muchas personas no pueden participar debido al gran número de barreras organizativas, estructurales, sociales etc. con las que se encuentran.

17. Ejemplos de esta discriminación los encontramos ya en la Atenas clásica, donde las mujeres, junto a los niños, los metecos y los esclavos fueron excluidos de la ciudadanía y, en consecuencia, de la posibilidad de poder participar en la A samblea, institución donde se tomaban las decisiones (Cortina, 1998).

18. Flecha (1995), enuncia y explica, a través de historias real es protagonizadas por personas comunes, los principios del aprendizaje dialógico.

19. En pocos años las mujeres han ocupado con fuerza su espacio como sujetos autónomos que emprenden una nueva vida en otros países, protagonizando importantes procesos migratorios (Bartolomé, 2002a). Un ejemplo de ello lo encontramos en M álaga, ciudad española donde la inmigración, concretamente la marroquí, ha sido desde su inicio femenina (El país digital. www.el pais.es).

20. Una revisión bibliográfica sobre la formación para la ciudadanía nos provee de un amplio abanico de programas que van desde aquellos centrados en los derechos y los deberes de los ciudadanos y ciudadanas (Knowing Your Welfare Rights \& Responsibilities in $M$ anitoba), hasta aquellos otros que mediante el apoderamiento de las mujeres buscan la igual dad de géneros (T he Fairy Tale Project) o aquellos que centrados en una identidad comunitaria pretenden conseguir la implicación de las personas en su comunidad (Building Strong N eighborhoods: A Study Circle Guide for Public D ialogue and Community Problem Solving). 
21. Previamente a la elaboración del programa de actividades se ha realizado un análisis de necesidades a partir de un análisis secundario y de un estudio exploratorio, que nos ha confirmado la fal ta de participación de las mujeres en esta ciudad.

22. Actual mente estamos real izando esta primera fase. L os primeros resultados de la misma se exponen en el apartado 9 de este trabajo.

23. El programa de formación para una ciudadanía activa se aplica en la Fundación A kwaba, O rganización No G ubernamental de desarrollo local ubicada en el barrio de CollblancTorrassa de $\mathrm{H}$ ospitalet de Llobregat que trabaja, principalmente, con mujeres.

24. El análisis de las entrevistas y las dinámicas participativas se ha realizado mediante el programa informático AT LAS-TI v.4.1. Se ha optado por utilizar este programa porque te permite establecer relaciones inter y entre categorias, documentos y codificaciones y, representarlas gráficamente mediante redes conceptuales.

25. En las relaciones entre las diferentes categorías de análisis, la letra G nos indica "es parte de..." y la letra R "está asociada con..."

26. N o se incluye en los resultados de esta categoría de análisis a las tres mujeres españolas que participan en la experiencia.

27. N o se incluye dentro de este resultado a las tres mujeres españolas que participan en la experiencia.

28. Espín (2002), realiza un análisis crítico sobre las dicotomías espacio privado y público / igual dad y diferencia, dicotomías que tradicional mente han estado presentes en la lucha de las mujeres por conseguir la plena ciudadanía.

29. Las actividades están pensadas para que el grupo se organice en torno a las necesidades que emanan de las propias personas que lo integran.

\section{Bibliografía}

Arendt, H . (1993). La condición humana. Barcelona: Paidós.

Banks, J. A. (1997). Educating citizensin a multicultural society. N ew York: Teachers College Press.

Bárcena, F. (1997). El oficio dela ciudadanía. Introducción a la educación política. Barcelona: Paidós.

Bartolomé, M . (2002a). El reto de las migraciones en sociedades multiculturales desde la perspectiva de la educación. En C. M orano (Ed.). Cuestiones emergentes en el diálogo Fe Cultura. Salamanca: U niversidad Pontificia de Salamanca y Cátedra Pedro Póveda.

Bartolomé, M . (2002b). Educar para una ciudadanía intercultural. En M . Bartolomé (Ed.), Identidad y ciudadanía: U n reto a la educación intercultural. M adrid: N arcea.

Bartolomé, M. y C abrera, F. (2000). N uevas tendencias en la evaluación de programas de educación multicultural, Revista de Investigación Educativa, 19(2), 463-479.

Bartolomé, M ., Cabrera, F., Espín, J.V., M arín, M .A. y Rodríguez, M . (1999). Diversidad y Multiculturalidad. Revista de Investigación Educativa, 17(2), 227-320.

Bartolomé, M ., Cabrera, F., D el Campo, J., Espín, J.V., M arín, M .A., Rodríguez, M ., Sandín, M .P y Sabariego, M . (2001). La construcción dela identidad en contextos multiculturales. $M$ adrid: CIDE.

Bartolomé, M. (coord.), Folgueiras, P., M assot, I ., Sabariego, M . y Sandín, M .P. (2003). ¿Construimos Europa? El sentimiento de pertenencia desde una pedagogía de la inclusión. $M$ adrid: $M$ inisterio de Educación. En prensa

Bauman, Z. (2001). Community. Cambridge: Polity Press.

Benhabib, S. (2000). D iversitat cultural, igualtat democràtica. Valencia: Tandem Edicions.

Bilbeny, N . (1995). Europa después de Sarajevo: Claves éticas y políticas dela ciudadanía europea. Barcelona: D estino.

Borja, J., N ello, O . y Vallès, J.M . (1998). La ciutat del futur, el futur delesciutats. Barcelona: Fundació Campanals. 
Bourdieu, P. (1984). D istinction: A social critique of thejudgment of taste. Cambridge, M A: $\mathrm{H}$ arvard U niversity Press.

Cabrera, F. (2002). H acia una nueva concepción de la ciudadanía en una sociedad multicultural. En M. Bartolomé (Ed.), I dentidad y ciudadanía: Un reto a la educación intercultural. M adrid: N arcea.

Camps, V. (1994). Los valores dela educación. M adrid: Anaya.

Carr, W., y Kemmis, S. (1988). Teoría crítica de la enseñanza. La investigación acción en la formación de profesorado. Barcelona: M artínez Roca.

Cortina, A. (1998). Ciudadanos del mundo. H acia una teoría dela ciudadanía. M adrid: Alianza Editorial, S.A.

Courtney, S. (1992). W hy adults learn: Towards a theory of participation in adult education. London: Routledge.

CREA (1995). Participación y no participación en educación de personasadultas. Informe de Investigación. Universidad de Barcelona: D ocumento policopiado.

Cruz, M . (1998). Tolerancia o barbarie. Barcelona: G edisa.

D el Campo, J. (2002). La gestión positiva del conflicto: Un camino para la convivencia intercultural. En M. Bartolomé (Ed.), I dentidad y ciudadanía: U n reto a la educación intercultural. M adrid: N arcea.

El País digital. Se puede encontrar en www.el pais.es

Espín, J.V. (1993). D iferencias de género en la intervención educativa: Un tema vigente en investigación. Revista del nvestigación Educativa, 23, 48-63.

Espín, J. V. (2002). Educación, ciudadanía y género. En M . Bartolomé (Ed.), I dentidad y ciudadanía: U n reto a la educación intercultural. M adrid: N arcea.

Etzioni, A. (2000). Thethird way to a good society. London: D emos.

Flecha, R. (1997). Compartiendo palabras. El aprendizaje de las personas adultasa traves del diálogo. Barcelona: Paidós.

Freire, P. (1993). Pedagogía de la esperanza. M adrid: Siglo XXI.

Freire, P. (1997). A la sombra de esteárbol. Barcelona: El Roure.

Folgueiras, P. y Sandín, M .P. (2003). La introducción de la dimensión europea en el curriculum de la Educación Secundaria O bligatoria: Una experiencia de evaluación participativa. En prensa.

Frazer, E. (1999). The problems of communitariam politics. N ew York-O xford: U niversity Press.

Gaventa, J., Valderrama, C. (1999). Participation, citizenship and local governance. Se puede encontrar en www.worldbank.org/wbi/communityempowerment/Resources/M odule_7.html

H abermas, J. (1991). Teoría de la acción comunicativa I. M adrid: Taurus.

H abermas, J. (1994). Three normative models of democracy. Constellations, 1(1), 1-10.

$\mathrm{H}$ abermas, J. (1998). I dentidades nacionales y postnacionales. M adrid: Editorial Tecnos, S.A.

H eater, D. (1999). What is citizenship? M alden, M A: Polity Press.

H enderson, P. y Salmon, H . (1998). Sign posts to local democracy. London: Warwick Business School.

H erbert, Y. (1997). Citizenship education: Towards a pedagogy of social participation and identity formation. Canadian Ethnic Studies/Études Ethniquesau Canada, 29(1), 82-96.

Kymlicka, W. (1996). Ciudadanía multicultural. Barcelona: Paidós.

Lister, R. (1997). Citizenship: Feminist perspectives. London: M acmillan Press LT D.

Luque D ominguez, P. A. (1995). Espacios educativos. Sobrela participación y la transformación social. Barcelona: EU B.

M aalouf, A. (1999). Lesidentitats quematen. Barcelona: La Campana.

M archioni (1999). Comunidad, participación y desarrollo. Teoría y metodología de la intervención comunitaria. M adrid: Editorial Popular.

M arín, M . A. (2002). La construcción de la identidad en la época de la mundialización y los nacionalismos. En M . Bartolomé(Ed.), Identidad y ciudadanía: U n reto a la educación intercultural. M adrid: N arcea. 
M arín, M .A. (coord.), Folgueiras, P., Sabariego, M . y Vilà, R.(2002). Buenas prácticas de educación intercultural. Informe de investigación. U niversidad de Barcelona: D ocumento policopiado.

M arshall, T. H . (1950). Citizenship and social class and other essays. C ambridge: Cambridge University Press.

M assot, M. I. (2001). Jóvenes entreculturas: La construcción dela identidad en contextos multiculturales. Bilbao: D esclée D e Brouwer.

Pagé, M . (1997). Pluralistic citizenship: A references for citizenship education. Canadian Ethnic Studies/ÉtudesEthniques au Canada, 29(2), 23-31.

Panikkar, R. (2000). El espíritu de la política: homo politicus. Barcelona: Península.

Pateman, C. (1970). Participation and democracy theory. Cambridge: Cambridge University Press.

Pateman, C. (1988). T hesexual contract. Cambridge: Polity Press.

Payá, M . (1997). Educación en valores para una sociedad abierta y plural. Bilbao: D esclée De Brouwer.

Pérez Ledesma, M . (Ed.) (2000). Ciudadanía y democracia. M adrid: Editorial Pablo I glesias.

Pérez Ledesma, M . (2000). Ciudadanos y ciudadanía: Un análisis introductorio. En M. Pérez Ledesma (Ed.), Ciudadanía y democracia (pp. 11-12). M adrid: Pablo Iglesias.

Rimmerman, C. A. (1997). The new citizenship. O xford: Westviewpress.

Rodríguez, M . (2002). Respuestas educativas al desarrollo de la identidad europea. En M . Bartolomé(Ed.), I dentidad y ciudadanía: Un reto a la educación intercultural. M adrid: Narcea.

Sabariego Puig, M . (2001). L'educació intercultural antelos retos del siglo XXI. Bilbao: D esclée DeBrouwer.

Sandín, M . P. (2003). Investigación cualitativa en educación. Fundamentos y tradiciones. M adrid: $\mathrm{McG}$ raw-H ill.

Schugurensky, D . (2001). Enlightenment and engagement in adult education for democratic citizenship: Lesson from the Citizen's Forum and theParticipatory Budget. C ánada:

D ocumento policopiado presentado en la C onference of C anadian Association for the Study of Adult Education.

Siim, B. (2000). G ender and citizenship. Cambridge: University Press.

Smith, J. y T he Social Planning C ouncil of Brandon (2001). Knowing your welfarerights \& responsibilities in $\mathrm{M}$ anitoba. Se puede encontrar en http://www.nald.ca/fulltext/guide/guide.pdf

Tam, H. (1998). Communitarism: A new agenda for politicsand citizenship. London: M acmillan Press LTD .

Taylor, CH . (1994). La ética dela autenticidad. Barcelona: Paidós.

The Calgary John H oward Society Literacy Program (2001). T hefairy taleproject. Calgary: The C algary John H oward Society Literacy Program.

Thibaut, C. (1998). La vindicación de ciudadano. Un sujeto reflexi vo en una soci edad compleja. Barcelona: Paidós.

Topsfield Foundation, Inc. (1998). Building strong neighborhoods. A study circleguide for public dialogue and community problem solving. Se puede encontrar en: http://www.studycircles.org/pdf/ampu.pdf

Toren, N. (1999). Women and immigrants: Strangers in a strange land. Gender Issues, 17(1), 76-95.

Trilla, J y N ovella, A.(2001) Educación y participación social de la infancia. Revista Iberomaericana deE ducación. 26, 137-164.

U rgenson, C. y Kember, M . (1997). Women and social policy. London: M acmillan Press.

U sher, R., Bryant, I. y Johnston, R. (1997). Adult education and the postmodern challenge: Learning beyond the limits. London: Routledge. 
Villasante, T.R., M ontañés, M . y M artí, J. (Ed.). (2000). La investigación social participativa. Construyendo ciudadanía/1. España: El viejo topo.

Vives, J. (9 de junio 2002). M ataró y el colectivo musulmán firman un pacto de convivencia. Barcelona: La Vanguardia (pp. 45).

Walzer, M . (1998). Tratado sobre la tolerancia. Barcelona: Paidós. 
46 Encounters/Encuentros/Rencontres 